\title{
Randomized Isomorphic Dvoretzky Theorem
}

\author{
Alexander LITVAK ${ }^{1} \quad$ Piotr MANKIEWICZ ${ }^{2}$ \\ Nicole TOMCZAK-JAEGERMANN ${ }^{3}$
}

\author{
Department of Mathematical and Statistical Sciences, University of Alberta, Edmonton, Alberta, \\ Canada T6G 2G1 alexandr@math.ualberta.ca \\ Institute of Mathematics, Polish Academy of Sciences, P.B. 137; 00-950 Warsaw, Poland \\ piotr@impan.gov.pl \\ Department of Mathematical and Statistical Sciences, University of Alberta, Edmonton, Alberta, \\ Canada T6G 2G1 nicole@ellpspace.math.ualberta.ca
}

\begin{abstract}
Let $K$ be a symmetric convex body in $\mathbb{R}^{N}$ for which $B_{2}^{N}$ is the ellipsoid of minimal volume. We provide estimates for the geometric distance of a "typical" rank $n$ projection of $K$ to $B_{2}^{n}$, for $1 \leq n<N$. Known examples show that the resulting estimates are optimal (up to numerical constants) even for the Banach-Mazur distance.
\end{abstract}

\section{Résumé \\ French title here}

Soit $K$ un corps convexe symétrique de $\mathbb{R}^{N}$ dont l'ellipsoïde de volume minimal le contenant est la boule euclidienne $B_{2}^{N}$. Nous estimons la distance géométrique de projections "typiques" de rang $n$ de $K$ à la boule $B_{2}^{n}$ pour tout $n \in\{1, \ldots, N-1\}$ (i.e. nous prouvons qu'il en existe une vaste proportion au sens de la mesure de Haar sur la grassmanienne). Des exemples bien connus permettent de dire que ces estimations sont optimales (à des constantes numériques près), même pour la distance de Banach-Mazur.

Précédemment, des résultats sur l'existence de projections orthogonales de $K$ (ou d'un vaste ensemble de telles projections si l'on ajoute une restriction sur leur rang) avec un bon contrôle de la distance à la boule euclidienne ont été prouvés dans [11] et [13], et dans un cadre gaussien dans [4] et [3]. Dans cette note, nous donnons une preuve unifiée de l'existence de projections orthogonales "typiques" pour toutes les valeurs de la dimension $n$.

Comparés aux précédents arguments connus, notre preuve repose sur deux nouveaux ingrédients. Tout d'abord, nous utilisons un résultat de [7] qui permet de réduire l'étude du rayon d'une boule euclidienne inscrite dans des projections aléatoires d'un corps convexe à la même question posée pour une boule euclidienne inscrite dans une section fixée du corps convexe. Le second ingrédient est un résultat de Vershynin [15] qui nous permet de trouver un sous-ensemble de points de contact équivalent à un système orthonormal.

\footnotetext{
${ }^{1}$ This author is partially supported by France-Israel Arc-en-Ciel exchange.

${ }^{2}$ The research of this author is partially supported by the grant KBN 2 P03A 01319.

${ }^{3}$ This author holds Canada Research Chair in Mathematics.
} 
The main result of this note is Theorem 1 below, which generalizes various extensions of Dvoretzky's theorem proved by Milman and Schechtman in [11], [13] (see also [3], [4], [6]). It provides estimates for radii of Euclidean balls inscribed and superscribed into a "typical" projection of a symmetric convex body $K$ with respect to the natural Euclidean structure induced by the ellipsoid of minimal volume. The existence (rather than "typicality") of projections with good control of the radii was proved in [13], and the result for "typical" projections was proved in [11] and [13] for a limited range of dimensions. In this note we provide a uniform proof for typical projections over the full range of dimensions.

Similarly as in [11] our proof follows a general line of [8], and compared with previous arguments it is based on two new ingredients. Firstly, we use a result from [7] which reduces the estimates for radii of a Euclidean ball inscribed in random projections of a convex body to a similar question inside any fixed section of the body $((*)$ below $)$. The second fact is a result of Vershynin [15] which provides a subset of contact points well equivalent to an orthonormal system ((**) below).

We also prove, in Proposition 4, a related simple optimization fact which states that the regular cube has the maximal Gaussian measure among all parallelopipeds inscribed in a Euclidean ball. This can be viewed as a complementary result to Gluskin's minimization theorem [1], which was also closely related to the Gluskin's lemma used in [13].

The example of the unit ball in $\ell_{1}^{n}$ shows that the estimates in Theorem 1 are optimal (up to numerical constants), even if the "geometric distance" is replaced by the smaller Banach-Mazur distance to the Euclidean ball.

Acknowledgement: The authors thank Roman Vershynin for pointing out to them his theorem from [15]. They are very grateful to Gideon Schechtman for his comments on the first version of this note. They are also very grateful to Olivier Guédon for translating the extended abstract into French.

For $N \geq 1$, by $\left\{e_{i}\right\}_{i=1}^{N}$ we denote the canonical vector basis in $\mathbb{R}^{N}$, equipped with the Euclidean norm $\|\cdot\|_{2}$. The unit ball with respect to $\|\cdot\|_{2}$ will be denoted by $B_{2}^{N}$, while $B_{\infty}^{N}$ will stand for the set of all $x=\sum t_{i} e_{i} \in \mathbb{R}^{N}$ such that $\left|t_{i}\right| \leq 1$ for all $1 \leq i \leq N$. $G_{N, k}$ will be the set of all $k$ dimensional subspaces of $\mathbb{R}^{N}$ equipped with the normalized Haar measure $\mu$. For $H \in G_{N, k}$, by $P_{H}$ we denote the orthogonal projection onto $H$. For a symmetric convex body $K$ in $\mathbb{R}^{N}$ we let $k^{*}(K)$ to be the maximal dimension $k$ such that for some constant $a>0$ we have

$$
\mu\left\{H \in G_{N, k} \mid a P_{H}\left(B_{2}^{N}\right) \subset P_{H}(K) \subset 4 a P_{H}\left(B_{2}^{N}\right)\right\} \geq 1-e^{-k},
$$

$c f .,[12],[9]$, and [7] (1.2) for the measure concentration used here.

Theorem 1 There exist constants $c, c^{\prime}>0$ such that for every $N \geq 1$ and every symmetric convex body $K$ in $\mathbb{R}^{N}$ for which the unit Euclidean ball $B_{2}^{N}$ is the ellipsoid of minimal volume containing $K$, and every $k^{*}(K) \leq n \leq N$, we have

$$
\mu\left\{H \in G_{N, n} \mid c \sqrt{\log (N / n) / N} P_{H} B_{2}^{N} \subset P_{H}(K) \subset c^{-1} \sqrt{n / N} P_{H} B_{2}^{N}\right\} \geq 1-e^{-c^{\prime} n} .
$$

The existence of an $H \in G_{N, n}$ satisfying the inclusions defining the set above was proved in [13], and Theorem 1 in the range $1 \leq n \leq c N / \log N$ was proved in [11] and [13] (see the Remark on page 162 of [13]). Here we provide a uniform proof of the measure estimates over the full range of dimensions.

Clearly, by the definition of $k^{*}, P_{H}(K)$ concentrates around $a P_{H}\left(B_{2}^{N}\right)$, for $1 \leq k \leq k^{*}(K)$. For $k \geq k^{*}(K)$, the fact that the right hand side inclusion holds with probability $\geq 1-e^{-c^{\prime} n}$ is the well known "shrinking" principle for the diameter of random projections of an arbitrary convex 
body, cf., [9] (also see [7], Proposition 4.3 for the formulation). The proof of the fact that the left hand side inclusion in the statement of Theorem 1 holds with probability $\geq 1-e^{-c^{\prime} n}$ depends in an essential way on a result from [7], Proposition 3.2 which yields:

$(*)$ There exists a constant $\bar{c}>0$ such that for an arbitrary symmetric convex body $K_{0}$ in an $N / 2$ dimensional subspace $F$ of $R^{N}$, and for every $n \leq N / 4$ we have

$$
\mu\left\{H \in G_{N, n} \mid P_{H}\left(K_{0}\right) \supset \bar{c} P_{H} B_{2}^{N}\right\} \geq \mu\left\{H \in G_{F, n} \mid P_{H}\left(K_{0}\right) \supset P_{H} B_{2}^{N}\right\}-e^{-\bar{c} n} .
$$

Therefore, for $n \leq \tilde{c} N$ setting $K_{0}=K \cap F$ the left hand side inclusion is a formal consequence (with different constants) of the following statement. The case $n>\tilde{c} N$ is trivial.

Proposition 2 There exist constants $c, c^{\prime} \geq 0$ and $0<\tilde{c}<1 / 4$ such that for every $K \subset \mathbb{R}^{N}$ as in Theorem 1 there exists a $N / 2$ dimensional subspace $F \subset \mathbb{R}^{N}$ such that for every $1 \leq k^{\prime} \leq \tilde{c} N$ we have

$$
\mu\left\{H \in G_{F, k^{\prime}} \mid P_{H}(K \cap F) \supset c \sqrt{\log \left(N / k^{\prime}\right) / N} P_{H} B_{2}^{N}\right\} \geq 1-e^{-c^{\prime} k^{\prime}} .
$$

The choice of a subspace $F$ will depend on the existence of contact points of the body $K$ and $B_{2}^{N}$ which are well equivalent to an orthonormal system. Recall that vectors $\left\{z_{i}\right\}_{i=1}^{m}$ in $\mathbb{R}^{m}$ are said to satisfy a lower $\ell_{2}$ estimate with constant $A_{1}>0$ and an upper $\ell_{2}$ estimate with constant $A_{2}$ provided that

$$
\left(1 / A_{1}\right)\left(\sum_{i=1}^{m} t_{i}^{2}\right)^{1 / 2} \leq\left\|\sum_{i=1}^{m} t_{i} z_{i}\right\|_{2} \leq A_{2}\left(\sum_{i=1}^{m} t_{i}^{2}\right)^{1 / 2}
$$

for all scalars $t_{1}, \ldots, t_{m}$.

Another crucial ingredient in our proof is a consequence of a recent result of Vershynin [15], Corollary 5.1, which can be formulated as follows:

$(* *)$ For every $K$ as in Theorem 1 there are $m \geq N / 2$ contact points $z_{1}, \ldots, z_{m}$ (i.e., $\left\|z_{i}\right\|_{K}=$ $\left\|z_{i}\right\|_{K^{0}}=\left\|z_{i}\right\|_{2}=1$ for $\left.i=1, \ldots, m\right)$ which satify lower and upper $\ell_{2}$ estimates with constants $A_{1}=A_{2}=C$, where $C>0$ is a numerical constant.

Thus Theorem 1 reduces to

Proposition 3 Let $m \geq 1$ and let $\left\{z_{i}\right\}_{i=1}^{m}$ in $\mathbb{R}^{m}$ satisfy lower and upper $\ell_{2}$ estimates with constants $A_{1}$ and $A_{2}$, respectively. There exist universal constants $c, c^{\prime}>0$ and $\tilde{c}=\tilde{c}\left(A_{1}, A_{2}\right)>0$ depending on $A_{1}, A_{2}$ only such that for every $1 \leq k^{\prime} \leq \tilde{c} m$ we have

$$
\mu\left\{H \in G_{m, k^{\prime}}\left|\sup _{1 \leq i \leq m}\right|\left(x, z_{i}\right) \mid \geq c \sqrt{\log \left(m / k^{\prime}\right) / m} \quad \text { for } x \in S^{m-1} \cap H\right\} \geq 1-e^{-c^{\prime} k^{\prime}} .
$$

Indeed, assuming the validity of Proposition 3, Proposition 2 can be proved for $F=\operatorname{span}\left[z_{i}\right]_{i=1}^{m}$. Identify this $F$ with $\mathbb{R}^{m}$. Since $K \cap F \supset \operatorname{conv}\left( \pm z_{i}\right)_{i=1}^{m}$, it is easy to see by duality that for a given $H \in G_{m, k^{\prime}}$ the condition $\sup _{1 \leq i \leq m}\left|\left(x, z_{i}\right)\right| \geq c \sqrt{\log \left(m / k^{\prime}\right) / m}$ for all $x \in S^{m-1} \cap H$ implies the inclusion $P_{H}(K \cap F) \supset c \sqrt{\log \left(N / k^{\prime}\right) / N} P_{H} B_{2}^{N}$. Thus Proposition 2 follows.

Proof of Proposition 3 Fix $1 \leq k \leq m$ and a partition $\left\{\sigma_{j}\right\}_{j=1}^{k}$ of $\{1,2, \ldots, m\}$ into mutually disjoint subsets with $[m / k] \leq\left|\sigma_{j}\right| \leq[m / k]+1$ for all $1 \leq j \leq k$. Define the norm $\|\cdot\|$ on $\mathbb{R}^{m}$ by

$$
\|x\|=(1 / k) \sum_{j=1}^{k} \sup _{i \in \sigma_{j}}\left|\left(x, z_{i}\right)\right| \text { for } x \in \mathbb{R}^{m} .
$$


We prove an estimate analogous to (1) for the norm $\|\cdot\| \|$, and we shall work in the Gaussian setting. Recall that a random vector $g \in \mathbb{R}^{m}$ of the form $g=\sum_{i=1}^{m} \tilde{\gamma}_{i} e_{i}$, where $\tilde{\gamma}_{i}$ are independent $N(0,1)$ distributed Gaussian variables, is called a standard Gaussian vector. The usual approach requires a lower estimate for the expected value $\mathbb{E}\|\mid g\|$, and an upper estimate for the Lipschitz constant of $\|\cdot\| \cdot \|$.

Let $\left\{z_{i}^{\prime}\right\}_{i=1}^{m}$ in $\mathbb{R}^{m}$ be the biorthogonal system to $\left\{z_{i}\right\}_{i=1}^{m}$; thus $\left\{z_{i}^{\prime}\right\}_{i=1}^{m}$ satisfies lower and upper $\ell_{2}$ estimates with constants $A_{2}$ and $A_{1}$, respectively. It easily follows from the lower $\ell_{2}$ estimate for $\left\{z_{i}^{\prime}\right\}$ 's that $\||\cdot| \mid$ satisfies the Lipschitz condition with respect to the Euclidean norm with constant $A_{2} / \sqrt{k}$. First we show that there is an absolute constant $c_{1}>0$ such that

$$
\mathbb{E}\left\||\| g| \geq\left(c_{1} / A_{1}\right) \sqrt{\log (1+m / k)}\right.
$$

Observe first that for any $1 \leq \ell \leq m$ we have

$$
\mathbb{E} \sup _{1 \leq i \leq \ell}\left|\left(g, z_{i}\right)\right| \geq\left(c_{1} / A_{1}\right) \sqrt{\log (\ell+1)}
$$

where $c_{1}>0$ is an absolute constant. Indeed, by Sudakov's minoration theorem (cf., e.g., [14], Theorems 5.5 and 5.6) we have

$$
\mathbb{E} \sup _{1 \leq i \leq \ell}\left|\left(g, z_{i}\right)\right| \geq c_{1} \sup _{\varepsilon>0} \varepsilon \sqrt{\log (1+N(\varepsilon))}
$$

where for a given $\varepsilon>0$, by $N(\varepsilon)$ we denote the covering number $N\left(\operatorname{conv}\left\{ \pm z_{i}\right\}_{1 \leq i \leq \ell}, \varepsilon B_{2}^{m}\right)$, i.e., the smallest number $N$ of balls $\varepsilon B_{2}^{m}$ which shifts can cover the set conv $\left\{ \pm z_{i}\right\}_{1 \leq i \leq \ell \text {. Since }}$ $\left\|z_{i}-z_{i^{\prime}}\right\| \geq \sqrt{2} / A_{1}$ for all $1 \leq i, i^{\prime} \leq \ell, i \neq i^{\prime}$, (4) immediately follows (with a different constant $c_{1}$ ). Then (3) follows by the definition (2) of $\|\mid \cdot\|$.

We give a few more details of Proposition 3. Let $k^{\prime}=\left[\alpha\left(c_{1} / A_{1} A_{2}\right)^{2} k\right]$, where $0<\alpha<1$ will be determined later. Let $\Gamma$ denote an $m \times k^{\prime}$ matrix with independent Gaussian $N(0,1)$ entries. We shall treat $\Gamma$ as the operator $\Gamma: \mathbb{R}^{k^{\prime}} \rightarrow \mathbb{R}^{m}$.

Fix $\beta>0$ sufficiently small. Since the Lipschitz constant of $\|\cdot\|$ is less than or equal to $A_{2} / \sqrt{k}$, by the standard Gaussian deviation inequality we get, for all $x \in S^{k^{\prime}-1}$

$$
\mathbb{P}\left\{\left|\left\|\Gamma x||-\mathbb{E}\left|\left\|\Gamma x|\|| \leq\left(\beta c_{1} / A_{1}\right) \sqrt{\log (1+k / m)}\right\} \geq 1-2(k / m)^{\left(\beta c_{1} / A_{1} A_{2}\right)^{2} k / 2} .\right.\right.\right.\right.
$$

Combining (3) with a standard $\varepsilon$-net argument (cf., e.g., [10]), and chosing a suitable $\varepsilon>0$, we obtain

$$
\mathbb{P}\left\{\|\Gamma x\| \geq\left(\beta c_{1} / 2 A_{1}\right) \sqrt{\log (1+m / k)} \text { for } x \in S^{k^{\prime}-1}\right\} \geq 1-e^{-k^{\prime}},
$$

provided that $k \leq \delta m<m$ and we chose $\alpha>0$ to satisfy $(3 / \varepsilon)^{-k^{\prime}} 2 \delta^{\left(\alpha c_{1} / A_{1} A_{2}\right)^{2} k / 2} \leq e^{-k^{\prime}}$.

Since $\sup _{1 \leq i \leq m}\left|\left(x, z_{i}\right)\right| \geq\|\mid\| x \|$ for all $x \in \mathbb{R}^{m}$, (1) follows from (5) by a standard argument.

Remark The proof above does not use the full strength of the lower $\ell_{2}$ estimate, but only a weaker fact that the vectors $\left\{z_{i}\right\}$ are well separated.

We next prove a related result which can also provide an alternative proof of (3). Namely, using Proposition 4 one easily shows that if $\left\{z_{i}\right\}$ are as in Proposition 3 then we have $\mathbb{P}\left\{\sup _{1<i<\ell}\left|\left(g, e_{i}\right)\right|>\right.$ $t\} \leq \mathbb{P}\left\{\sup _{1<i<\ell}\left|\left(g, z_{i}\right)\right|>t / A_{1}\right\}$, for all $t>0$. Proposition 4 is a natural maximization result which might be of an independent interest, and we provide a sketch of its proof. 
Proposition 4 Let $n \geq 1$ and let $g$ be a standard Gaussian vector in $\mathbb{R}^{n}$. Let $y_{1}, \ldots, y_{n} \in \mathbb{R}^{n}$ and let $Q=\sum_{i=1}^{n}\left[-y_{i}, y_{i}\right]$ be the parallelopiped generated by the $y_{i}$ 's. If $Q \subset \sqrt{n} B_{2}^{n}$ then for an arbitrary $t>0$ we have $\mathbb{P}\{g \in t Q\} \leq \mathbb{P}\left\{g \in t B_{\infty}^{n}\right\}$.

Proof It suffices to prove that for every $a>0$, the inclusion $Q \subset a B_{2}^{n}$ implies $\mathbb{P}\{g \in Q\} \leq \mathbb{P}\{g \in$ $\left.(a / \sqrt{n}) B_{\infty}^{n}\right\}$. To this end set $Q^{*}=\sum_{i=1}^{n-1}\left[-y_{i}, y_{i}\right]$. Clearly we may assume that $Q^{*} \subset \mathbb{R}^{n-1}=$ $\operatorname{span}\left[e_{i} \mid 1 \leq i \leq n-1\right]$.

Write $y_{n}$ in the form $y_{n}=v+t e_{n}$, where $v \in \mathbb{R}^{n-1}$. Replacing $y_{n}$ by $-y_{n}$ if needed we may assume that $t>0$. Let $y$ be any vertex of $Q^{*}$. By the parallelogram identity applied to $y$ and $y_{n}$, using the fact that $Q \subset a B_{2}^{n}$, we get $a^{2} \geq\left(\left\|y+y_{n}\right\|_{2}^{2}+\left\|y-y_{n}\right\|_{2}^{2}\right) / 2=\|y\|_{2}^{2}+\|v\|_{2}^{2}+t^{2}$. Hence $Q^{*} \subset \sqrt{a^{2}-t^{2}} B_{2}^{n-1}$.

For $k \geq 1$ by $\gamma_{k}$ denote the standard Gaussian measure on $\mathbb{R}^{k}$, so that we have $\gamma_{k}(B)=\mathbb{P}\{g \in$ $B\}$ for every Borel subset $B$ of $\mathbb{R}^{k}$. For $s \in \mathbb{R}$, let $\mathbb{R}_{s}^{n-1}=\mathbb{R}^{n-1}+s e_{n}$, and let $\gamma_{n-1, s}\left(B+s e_{n}\right)=$ $\gamma_{n-1}(B)$ for every Borel subset $B$ of $\mathbb{R}^{n-1}$. Observe that for $|s| \leq t$ we have $Q \cap \mathbb{R}_{s}^{n-1}=$ $Q^{*}+(s / t) y_{n}=\left(Q^{*}+s e_{n}\right)+(s / t) v$. Thus, by Anderson's inequality (cf., e.g., [5], Sec. 11, Th. 9), we have

$$
\gamma_{n-1, s}\left(Q \cap \mathbb{R}_{s}^{n-1}\right) \leq \gamma_{n-1, s}\left(Q^{*}+s e_{n}\right)=\gamma_{n-1}\left(Q^{*}\right) .
$$

The proof of the proposition will be completed by induction. For $n=1$ the statement is trivial. Assume the statement for $n-1$. Since $Q^{*} \subset \sqrt{a^{2}-t^{2}} B_{2}^{n-1}$, by the induction hypothesis and (6), for every $|s| \leq t$ we get $\gamma_{n-1, s}\left(Q \cap \mathbb{R}_{s}^{n-1}\right) \leq \gamma_{n-1}\left(b B_{\infty}^{n-1}\right)$, where $b=\sqrt{\left(a^{2}-t^{2}\right) /(n-1)}$. Thus

$$
\gamma_{n}(Q)=\int_{-t}^{t} \gamma_{n-1, s}\left(Q \cap \mathbb{R}_{s}^{n-1}\right) e^{-s^{2} / 2} \frac{d s}{\sqrt{2 \pi}} \leq(2 \pi)^{-n / 2} \int_{-t}^{t} e^{-s^{2} / 2} d s\left(\int_{-b}^{b} e^{-s^{2} / 2} d s\right)^{n-1}
$$

Consider the function $f(h)=\int_{0}^{h} e^{-s^{2} / 2} d s$ for $h \geq 0$ and note that $f$ is increasing while $f^{\prime}$ is decreasing. The inequality (7) can be expressed as

$$
\gamma_{n}(Q) \leq(2 / \pi)^{n / 2} f(a \cos \alpha) f^{n-1}((a \sin \alpha) / \sqrt{n-1})=: \Phi(\alpha),
$$

where $\alpha \in[0, \pi / 2]$ is defined by the equality $\cos \alpha=t / a$ (note that clearly $t \leq a$ ). Now, using the fact that the functions $f^{\prime}$ and $\cos \alpha$ are decreasing while the functions $f$ and $\sin \alpha$ are increasing we deduce that $\Phi^{\prime}$ has only one zero in $(0, \pi / 2)$, which is attained at the point $\beta$ satisfying $\sin \beta / \sqrt{n-1}=\cos \beta$. This yields $\sin \beta=\sqrt{(n-1) / n}$ and $\cos \beta=1 / \sqrt{n}$. Since $\Phi$ is nonnegative and $\Phi(0)=\Phi(\pi / 2)=0$ we infer that $\Phi$ attains its maximum at $\beta$. In view of (8) the proof is completed by observing that $\Phi(\beta)=\gamma_{n}\left((a / \sqrt{n}) B_{\infty}^{n}\right)$.

Remark The results of [11] and [13] were extended to the non-symmetric case by two different approaches in [3] and [6] (with the former paper giving randomness of projections for the limited range of ranks). In the present formulation Theorem 1 holds for nonsymmetric body $K$ and $n \leq \alpha N$ for some $0<\alpha<1$ (and hence for $n \leq \beta N$ for every $0<\beta<1$, with constants depending on $\beta$ ). Indeed, one can check that $(*)$ and $(* *)$ remain valid for nonsymmetric bodies. Our argument can be adopted to the nonsymmetric case by replacing $\left|\left(x, z_{i}\right)\right|$ with $\left(x, z_{i}\right) \vee 0$ in the definition of $\|\cdot\| \cdot$

Remark In [4] and [3] techniques developed in [2] were used to obtain an upper bound for the minimal Banach-Mazur distance of an $n$-dimensional section of an arbitrary $N$-dimensional convex body to the $n$-dimensional Euclidean ball, similar as in [11] and [13]. The same techniques may be used in our context as well. 


\section{References}

[1] E. D. Gluskin: Extremal properties of orthogonal parallelopipeds and their applications to the geometry of Banacj spaces; Math. USSR Sbornik, 64 (1989), 85-96.

[2] Y. Gordon: Some inequalities for Gaussian processes and applications; Israel J. of Math., 50 (1985), 265-289.

[3] Y. Gordon, O. Guédon \& M. Meyer: An isomorphic Dvoretzky theorem for convex bodies; Studia Math., 127 (1998), 191-200.

[4] O. GuÉdon, Gaussian version of a theorem of Milman and Schechtman; Positivity, 1 (1997), 1-5.

[5] M. A. Lifshits: Gaussian random functions. Mathematics and its Applications; 322. Kluwer Academic Publishers, Dordrecht, 1995.

[6] A. E. Litvak \& N. Tomczak-Jaegermann: Random aspects of high dimensional convex bodies. In: Geometric Aspects of Functional Analysis, Israeli Seminar, Lecture Notes in Math., 1795, Springer, 2000, pp. 169-191.

[7] P. Mankiewicz \& N. Tomczak-Jaegermann: Geometry of families of random projections of symmetric convex bodies; GAFA, Geom. and Funct. Anal., 11 (2001), 1282-1326.

[8] V. D. Milman, A new proof of the theorem of A. Dvoretzky on sections of convex bodies. Funct. Anal. Appl., 5 (1971), 28-37 (English translation).

[9] V. D. Milman: Surprising geometric phenomena in high dimensional convexity theory, Proceedings of II European Congress of Mathematicians, Budapest, 1996.

[10] V. D. Milman \& G. Schechtman: Finite-Dimensional Normed Spaces, Lecture Notes in Math., 1200, Springer, Berlin-New York, 1986.

[11] V. D. Milman \& G. Schechtman: An "isomorphic" version of Dvoretzky's theorem; C.R. Acad. Sci. Paris, Sér. I 321 (1995), 541-544.

[12] V. D. Milman \& G. Schechtman, Global versus local asymptotic theories of finite-dimensional normed spaces. Duke Math. J., 90 (1997), 73-93.

[13] V. D. Milman \& G. Schechtman: An "Isomorphic" Version of Dvoretzky's Theorem, II; Convex Geometric Analysis, MSRI Publ., Vol. 34, Cambridge University Press, 1998, 159-164.

[14] G. PISIER: The volume of convex bodies and Banach space geometry. Cambridge Univ. Press 1989.

[15] R. Vershynin: John's decomposition: selecting a large part; Israel J. of Math., 122 (2001), 253-277. 